\title{
Phraseologismen im Bereich der Sexualität Eine lexikographische Analyse an deutschen und polnischen Beispielen
}

\author{
Aleksandra LIDZBA, Krystian SUCHORAB
}

\begin{abstract}
Phraseology in terms of sexuality: a lexicographic analysis using German and Polish examples

People's sex life is very often, if not always, taboo in everyday life. The theming of this area of life is made possible by various linguistic means that allow one to speak about this content. Phraseological units also serve this purpose. The starting point of this article is the definition of phrasemes according to Burger (2015:11): "Firstly, they [phraseologisms] consist of more than one word; secondly, the words are not put together for this one occasion, but are combinations of words that we, as German speakers, know exactly in this combination (possibly with variants), similar to how we know German words as individual items". In addition, it is noteworthy that thanks to the characteristic of idiomaticity (cf. Fleischer 1982:30), this taboo is particularly reinforced.

At the center of our analysis are phrasemes related to sex life. The research material was taken from German and Polish dictionaries. The purpose of the presentation is to create a typology of thematic areas which are characterized with the help of phrasemes relating to sex life in German and Polish.

The article is based on the following definition of a taboo: "an unwritten law that forbids doing certain things based on certain beliefs within a society" (Duden 2015:1735).
\end{abstract}

Keywords: phrasemes, sex life, sexuality, sexual intercourse

DOI: doi.org/10.15452/StudiaGermanistica.2021.28.0002

\section{Einleitung}

In der Sprache werden alle Bereiche des menschlichen Lebens thematisiert. Einer der thematischen Bereiche ist die Sexualität. Diese Thematik wird aber selten, wenn überhaupt angesprochen, da das Sprechen über Sexualität zu den Tabuthemen gehört (vgl. Frietsch/Hanitzsch/John/Michaelis 2007; Knaut 2011; Schuch 2012; Rada 2013 u. a.). Aber im Sinne des Prinzips „Tabus sind da, um gebrochen zu werden!“ (URL 1) möchten wir uns auf die Problematik der Thematisierung der Sexualität im Lichte der deutschen und polnischen Phraseologie konzentrieren. Dank ihrer Merkmale erlauben nämlich Phraseologismen den Sprechern, etwas indirekt zu verbalisieren. Es handelt sich dabei v. a. um euphemistische Phraseologismen, mit deren Hilfe man einen Sachverhalt bzw. ein Thema mildernd zum Ausdruck bringen kann. 
In unserem Beitrag werden deutsche und polnische Phraseologismen untersucht, die zur Thematisierung des Geschlechtsverkehrs dienen und die im weiteren Sexualismen genannt werden. Sie wurden deutschen und polnischen lexikographischen Werken ${ }^{1}$ entnommen. Die Analyse hat zum Ziel, eine Typologie der thematischen Bereiche der auf den Geschlechtsverkehr Bezug nehmenden Phraseologismen zu erstellen und diese in beiden Sprachen zu vergleichen. Es handelt sich dabei um eine lexikographische Analyse. ${ }^{2}$ Dabei gehen wir von der „Phraseologie im weiteren Sinne“ nach Burger (2015:14) aus: „Die Menge derjenigen Phraseme, die die folgenden zwei Eigenschaften aufweisen, bildet den Bereich der Phraseologie im weiteren Sinne: (1) Polylexikalität - das Phrasem besteht aus mehr als einem Wort, (2) Festigkeit - wir kennen das Phrasem in genau dieser (oder einer sehr ähnlichen) Kombination von Wörtern, und es ist in der Sprachgemeinschaft - ähnlich wie ein Wort - gebräuchlich“. Daher berücksichtigen wir auch solche Einheiten, die nicht alle phraseologischen Merkmale erfüllen.

\section{Zum Wesen des Tabus}

Sexualität ist heute immer noch ein Thema, das man öffentlich nicht aufgreift bzw. nicht aufgreifen darf. Obwohl das Sexualleben ein untrennbarer Teil unseres Lebens ist, herrscht die Überzeugung, dass es sich nicht geziemt, darüber zu sprechen. Deswegen werden bestimmte Wörter bzw. Wortkonstruktionen tabuisiert. Dabei soll man von dem lateinischen Begriff „,verbum proprium“ ausgehen. Genau verba propria werden laut Dąbrowska tabuisiert. Sie schreibt darüber wie folgt (Dąbrowska 2013:12):

„Taka istniejąca nazwa jakiegoś zjawiska to nazwa wprost, czyli, używając terminu łacińskiego, verbum proprium. To właśnie verbum proprium jest objęte tabu, czyli stanowi wyraz, którego w danym kontekście nie chce się użyć, chociaż się go zna. [...] Jeśli objaśnieniami w tym opracowaniu są verba propria, to oznacza, że w definicjach pojawiać się muszą sformułowania, na które nałożone jest tabu językowe, a więc takie wyrazy, wyrażenia i zwroty, które są (mogą być) społecznie nie akceptowane. Nazwa wprost, stanowiąca określenie definiujące, może być więc wyrazem wulgarnym lub obscenicznym." ${ }^{3}$

Im Weiteren sollte man auf den Terminus „Tabu“ eingehen. Im Duden wird er folgendermaßen erklärt: „1. Verbot, bestimmte Handlungen auszuführen, besonders geheiligte Personen oder Gegenstände zu berühren, anzublicken, zu nennen, bestimmte Speisen zu genießen. 2. ungeschriebenes Gesetz, das aufgrund bestimmter Anschauungen innerhalb einer Gesellschaft verbietet, bestimmte Dinge zu tun“ (URL 2). Darüber hinaus soll man auch darauf hinweisen, dass ,man unter dem Begriff ,Tabu' (engl. taboo) im Allgemeinen ein unausgesprochenes, in einer Kultur allgemeingültiges Verbot versteht. Der starke Gegensatz zu normativen Gesetzen besteht darin, dass ein Tabu kaum hinterfragt, ungern diskutiert und deswegen selten genau umrissen ist" (URL 3). Lauenstein gibt demnach eine neutrale Erklärung des Begriffs „Tabu“ an: „Die neutrale Definition von Tabu ist: ein soziales Gebot, an das man sich halten soll.“(URL 1) Nickel (2003:77) schreibt über das Tabu folgendermaßen: „Der Begriff Tabu meint entweder ein einfaches Verbot oder die Weigerung, über bestimmte Dinge zu sprechen, nachzudenken oder zu diskutieren“. Tabu wird aber von Dąbrowska

Siehe Quellen am Ende des Beitrags.

2 Die Analyse des Vorkommens der von uns analysierten Einheiten im gegenwärtigen Deutsch und Polnisch ist nicht Gegenstand unseres Beitrags.

3 Dieser Name eines Phänomens ist ein direkter Name, also aus dem Lateinischen verbum proprium. Genau verbum proprium wird tabuisiert. Es ist ein Wort, das man in einem bestimmten Kontext nicht verwenden möchte, obwohl es [dem Sprechenden - K. S.] bekannt ist. [...] Wenn die Erklärungen in dieser Arbeit verba propria sind, bedeutet das, dass in ihren Definitionen Ausdrücke vorkommen müssen, die sprachlich tabuisiert werden, also solche Wörter, Phrasen und Ausdrücke, die gesellschaftlich unakzeptabel sind bzw. sein können. Der direkte Name, der die definitorische Bezeichnung ist, kann ein vulgäres oder obszönes Wort sein. - Übersetzung der polnischsprachigen Zitate ins Deutsche hier und im ganzen Text K. S. 
(2013:11) breiter verstanden. Sie weist nämlich darauf hin, dass es bestimmte sprachliche Mittel gibt, um über Tabus sprechen zu können:

„[...] współcześnie tabu oznacza jakikolwiek zakaz społeczny, zakaz ,w ogóle‘ Mówiąc o tabu językowym myśli się o społecznym zakazie wypowiadania pewnych wyrazów. A skoro pewnych wyrazów wypowiedzieć z jakiegoś powodu nie wolno, a o danym zjawisku mówić trzeba lub ma się na to ochotę, w miejsce zakazanych stosuje się określenia zastępcze, nie łamiące tabu, czyli właśnie eufemizmy."4

„Das Tabu“ ist also ein ungeschriebenes Verbot, dessen Brechen als unmoralisch und als Verstoß gegen die Konvention gilt. Eine bestimmte Sache wird ungern thematisiert und die Berührung dieses Thema ruft oft eine Erregung bei anderen hervor.

\section{Sprachliche Mittel zur Thematisierung der Sexualität im Bereich des Ge- schlechtsverkehrs}

Themen, die mit Tabu belegt sind, werden mithilfe von verschiedenen sprachlichen Mitteln aufgegriffen. Laut Dąbrowska kann man u. a. solche Sprachmittel auflisten: umgangssprachliche Ausdrücke (vgl. Dąbrowska 2013:85 f.; 88), fachliche Termini (ebd.:86), Individualismen (ebd.:85, 87, 90), offizielle Ausdrücke (ebd.:88), Okkasionalismen (ebd.:88), Euphemismen (ebd.:89), Periphrasen (ebd.:89) umgangssprachliche vulgäre Euphemismen (ebd.:89), lateinische Lehnwörter (ebd.:92) und Metaphern (ebd.:93). Rada (2013:91) unterscheidet dagegen: Simplizia und Wortbildungskonstruktionen (Komposita, Ableitungen (Präfigierungen, Suffigierungen, Konversionen), Kurzwörter, Kontaminationen und Reduplikationen), aus denen okkasionelle und usuelle Euphemismen gebildet werden.

Im Mittelpunkt der vorliegenden Studie stehen „Euphemismen“ bzw. „euphemistische Phraseologismen“.

\subsection{Phraseologismen}

Der Terminus „Phraseologismus“ wird vom Burger folgendermaßen definiert: „Erstens bestehen sie [Phraseologismen - A. L., K. S.] aus mehr als einem Wort, zweitens sind die Wörter nicht für dieses eine Mal zusammengestellt, sondern es handelt sich um Kombinationen von Wörtern, die uns als Deutschsprechenden genau in dieser Kombination (eventuell mit Varianten) bekannt sind, ähnlich wie wir die deutschen Wörter (als einzelne) kennen“" (Burger 2015:11).

In der Forschungsliteratur zur Phraseologie werden bestimmte Merkmale des „Phraseologismus“ angegeben, und zwar Idiomatizität, Stabilität (auch Festigkeit genannt), Lexikalisierung, Reproduzierbarkeit und Polylexikalität. Diese werden im Folgenden erklärt:

- Idiomatizität - „das Fehlen eines derivationell-semantischen Zusammenhangs zwischen dem semantischen Äquivalent eines Gliedes des Verbandes und den anderen Bedeutungen desselben Wortes“ (Fleischer 1997:30).

- Stabilität - die Festigkeit „,der phraseologischen Wortgruppen ist nicht allzu absolut zu nehmen und geradezu eine Herausforderung zum kreativen Aufbrechen und Spiel mit den festen Strukturen“ (Palm 1995:29). Burger erwähnt bei Stabilität auch den Begriff Variation und versteht darunter, dass „für viele Phraseme es nicht eine, vollständig fixierte Nennform gibt [...], sondern zwei oder mehrere sehr ähnliche Varianten“" (Burger 2015:22).

Heute bedeutet Tabu irgendein gesellschaftliches Verbot, ein Verbot „im Allgemeinen“. Wenn man über Tabu spricht, denkt man an ein gesellschaftliches Verbot, bestimmte Wörter auszusprechen. Und wenn einige Wörter aus irgendwelchen Gründen nicht geäußert werden dürfen, aber man braucht sie oder möchte darüber sprechen, werden an der Stelle der verbotenen Wörter Ersatzeinheiten gebraucht, die das Tabu nicht brechen. Das sind eben Euphemismen. 
- Lexikalisierung - „Die Lexikalisierung der syntaktischen Konstruktion bedeutet, da $\beta$ sie nicht mehr nach einem syntaktischen Strukturmodell in der Äußerung ,produziert‘, sondern daß sie als ,fertige“ lexikalische Einheit ,reproduziert" wird“" (Fleischer 1997:63).

Reproduzierbarkeit - „Die ,Reproduzierbarkeit“ der ,fertigen“ Konstruktion bei ihrer Verwendung in der Kommunikation, das heißt auch die in diesen Fällen erfolgte Speicherung als lexikalische Einheit, nicht nur die Tendenz zur Speicherung, wird nicht selten als das entscheidende Kriterium für die Zuordnung einer Wortverbindung zu den Phraseologismen überhaupt betrachtet" (ebd.).

Polylexikalität - Burger schreibt, dass sie ,relativ unproblematisch definierbar ist. Ob ein Ausdruck mehrere Wörter umfasst, ist in der Regel leicht entscheidbar, sofern man sich darauf einigen kann, was ein Wort ist. [...] die maximale Ausdrehung eines Phrasems ist üblicherweise nicht lexikalisch, sondern syntaktisch festgelegt: der Satz gilt als die obere Grenze phraseologischer Wortverbindungen“" (Burger 2015:15).

\subsection{Euphemismen}

Die Aufgabe von „Euphemismen“ ist es, bestimmte Ausdrücke mit anderen positiveren sprachlichen Einheiten zu ersetzen. Im Duden (URL 4) lässt sich eine folgende Definition des „Euphemismus“ finden: „,beschönigende, verhüllende, mildernde Umschreibung für ein anstößiges oder unangenehmes Wort".

Rada erklärt dagegen den Begriff „Euphemismus“ in Bezug auf den Terminus „Tabu“. Ihre Definition lautet:

„Sprachliche Tabus habe ich als sprachliche Norm, den Euphemismus als sprachliche Reaktion auf diese definiert. Der Euphemismus gilt als eine gesellschaftliche bedingte und verlangte Ausdrucksweise, die den als anstößig (weil tabu) empfundenen Aspekt eines Wortes bzw. Begriffes mildernd formuliert." (Rada 2013:65)

Die polnische Definition dieses Begriffs schlägt Dąbrowska (2013:11) vor:

„Eufemizm jest zatem zjawiskiem językowym, którego przyczyny pojawiania się są pozajęzykowe. Jest on zastępczą nazwą jakiegoś zjawiska, stosowaną zamiast nazwy wprost, która nie może być użyta, ponieważ skojarzenia z nią związane są negatywne. Określenie eufemistyczne powinno natomiast wzbudzać pozytywne (lub neutralne) konotacje." 5

Die Typologie der Euphemismen basiert sehr oft auf ihren Formen und Funktionen. Eine Typologie in Bezug auf Form bzw. Mittel, auf denen Euphemismen basieren, entwirft u. a. Dąbrowska (1994:333-366, zit. nach Waśniewska 2017:85, Hervorhebung im Original), die die folgenden Mittel unterscheidet: ,metonimia $[\ldots]$, peryfraza $[\ldots]$, antonomazja $[\ldots]$, litota $[\ldots]$, ironia $[\ldots]$, aposjope$z a[\ldots]$, aluzja [...], niedomówienie [...]." ${ }^{\text {6 }}$ Eine andere Typologie bezieht sich auf Funktionen der Euphemismen. Die Hauptfunktionen werden u. a. von Nefedova (vgl. 2019:288) unterschieden. Das sind Verhüllung und Verschleierung. Im Lichte dessen kann man auf die Zweiteilung der Euphemismen im Rahmen der funktionellen Typologie hinweisen. Das sind laut Bąk (2012:33) ,verhüllende“ und ,verschleiernde“ Ausdrücke. Luchtenberg (1985:152, zit. nach Bąk 2012:33) „,[rechnet - K. S.] zu den Kriterien der Abgrenzung [...] primär die Erkenntnis, dass die verhüllenden Euphemismen als gesellschaftlich anerkannt gelten und zum allgemeinen Wortschatz gehören“. Dagegen ,[soll] der verschleiernde Euphemismus [...] idealerweise eine Ad-hoc-Bildung sein, an der die kommunikative Absicht zu euphemisieren, nicht bzw. nicht auf den ersten Blick erkennbar sein sollte“ (ebd., vgl. auch Rada 20013; Forster 2009). Laut solcher Abgrenzung unterscheidet Rada (2013:91) zwischen

5 Euphemismus ist daher ein sprachliches Phänomen, dessen Erscheinungsursachen außersprachlich sind. Er ist ein Ersatzname eines Phänomens, der statt des direkten Namens, der nicht verwendet werden kann, weil er negativ assoziiert wird, gebraucht wird. Der euphemistische Ausdruck sollte jedoch positive (oder neutrale) Konnotationen hervorrufen.

6 Metonymie [...], Periphrase [...], Antonomasie, [...], Litotes [...], Ironie [...], Aposiopese [...], Anspielung [...] Unklarheit bzw. Verschweigen 
usuellen und okkasionellen Euphemismen. Überdies nennt sie bestimmte sprachliche Einheiten, die zu den okkasionellen und usuellen Euphemismen gehören.

Dabei soll man auch betonen, dass ,die verhüllenden Euphemismen unbewusste Reaktionen auf Tabuisierungen sind, während die Verschleierung mit der bewussten Täuschungsabsicht sind oder der Intention der Beeinflussung einhergeht“ (vgl. Dietl 1996; Forster 2009:74, zit nach. Bąk 2012:33).

Im Lichte dessen kann man darauf hinweisen, dass „Euphemismen“ dem Ausdruck der Höflichkeit dienen, ,weil sie als Meliorativa negativ konnotierte Sachverhalte durch positive Ausdrucksweise aufwerten und direkte pejorative Bezeichnungen ersetzen“ (Nefedova 2019:288). Nefedova (ebd.) schreibt auch, dass man „unter Euphemismus [...] einen sprachlichen Ausdruck [versteht - K. S.], der eine Person, eine Personengruppe, einen Gegenstand oder einen Sachverhalt beschönigend, mildernd oder in verschleiernder Absicht benennt".

Im Fokus dieses Beitrags stehen ,euphemistische Phraseologismen“. Darunter kann man Wortverbindungen verstehen, die die Funktionen der „Euphemismen“ erfüllen. Nevedova (2019: 288 f.) legt diesen Terminus folgendermaßen aus: „Euphemistische Phraseologismen sind lexikalisierte Wortverbindungen mit beschönigender Bedeutung, die verhüllende Funktion haben“. Es gilt auch die Worte von Bąk zu erwähnen, der zu ,euphemistischen Phraseologismen“ und ihrer Charakteristik wie folgt schreibt: „Das Merkmal der Lexikalisierung trifft auf alle usuellen Euphemismen zu, die Polylexikalistät ist für alle Euphemismen in Form einer Wortverbindung charakteristisch, die usuellen Euphemismen in Form einer Wortverbindung sind als euphemistische Phraseologismen [...]“ (Bąk 2012:93). Dabei kann man auch nach Dietz (vgl. 1999:263) betonen, dass solche Einheiten darin bestehen, dass sie mithilfe von Umschreibung einen negativ assoziierten bzw. konnotierten Sachverhalt/Umstand verhüllen, um den Gesprächspartnern zu schonen. Fleischer (1982:203) hebt auch hervor, dass ,euphemistische Phraseologismen [...] zum Teil als ,gehoben“ gekennzeichnet [sind - K. S.]“. Das heißt, dass sie übliche Sachverhalte auf solche Art und Weise zum Ausdruck bringen, dass sie mehr poetisch bzw. schöpfend klingen.

Im Weiteren werden feste Wortverbindungen im Deutschen und Polnischen präsentiert, die das Sexualleben, genauer den Geschlechtsverkehr, euphemistisch thematisieren.

\section{Analyse des Materials}

Das gesamte Material wurde lexikographischen Werken beider Sprachen entnommen. Die deutschen Quellen waren die folgenden Wörterbücher: ,Wörterbuch der deutschen Umgangssprache“ von Küpper (1966) und ,Sex im Volksmund“ von Borneman (2013). Polnische Beispiele wurden aus folgenden Werken exzerpiert: ,Słownik eufemizmów polskich, czyli w rzeczy mocno, w sposobie łagodnie“ von Dąbrowska (2013), ,Słownik polskich przekleństw i wulgaryzmów‘ von Grochowski (1995) und ,Mały słownik erotyzmów polskich“ von Kociemba-Żulicka (2011). Aufgrund des umfangreichen Materials werden nur repräsentative Beispiele präsentiert.

Die Arbeit hat zum Ziel, einen Überblick über phraseologische Wendungen anzubieten, die sich auf Sexualleben beziehen, und darauf hinzuweisen, dass sowohl deutsche als auch polnische Sprache den Sprechern ermöglicht, über verschiedene Geschlechtsverkehrstypen / -arten zu diskutieren. Der Beitrag gilt als eine Einführung in weitere Forschung, in der sprachliche Mittel zur Thematisierung von anderen thematischen Bereichen des genannten Feldes genauer untersucht und präsentiert werden.

Die folgende Tabelle stellt deutsche und polnische Beispiele dar, die verschiedene Geschlechtsverkehrstypen auf idiomatische Art und Weise beschreiben. 


\begin{tabular}{|c|c|}
\hline Deutsch & Polnisch \\
\hline \multicolumn{2}{|c|}{ 1. Bezeichnungen für } \\
\hline \multicolumn{2}{|c|}{ a. Masturbieren } \\
\hline $\begin{array}{l}\text { sexueller Kurzschluß - ,Onanie‘ (WU2:175) } \\
\text { ein Händedruck unter Männern - ,gegenseitige } \\
\text { Masturbation zwischen Männern‘ (EB:83) }\end{array}$ & $\begin{array}{l}\text { jezuicki koncert na cudze ręce-, doprowadzenie } \\
\text { partnera do orgazmu przez pieszczenie jego genita- } \\
\text { liów; eufemizm, okazjonalizm“ (MSEP:118) } \\
\text { gra w kulki-,masturbacja; potoczne' (MSEP:159) } \\
\text { marszczyć Freda - ,o mężczyźnie: masturbować się; } \\
\text { potoczne, rubaszne' (MSEP:159) }\end{array}$ \\
\hline \multicolumn{2}{|c|}{ b. Geschlechtsverkehr } \\
\hline & $\begin{array}{l}\text { kanapowe ekscesy ,lekceważąco o stosunku } \\
\text { seksualnym; okazjonalizm’ (MSEP:118) } \\
\text { szybki numerek ,pogardliwie o stosunku seksualnym } \\
\text { pozbawionym gry wstępnej; eufemizm wulgarny’ } \\
\text { (MSEP:140) } \\
\text { tradycyjne metody osiagnięcia błogosławionego } \\
\text { stanu ,stosunek płciowy. Okazjonalizm zawierający } \\
\text { eufemizm - błogosławiony stan' (SEP:103) } \\
\text { zabawa z wenera ,stosunek płciowy. Staropolskie’ } \\
\text { (SEP:105) } \\
\text { histeryczne psie zwarcie, gwałtowny stosunek seksu- } \\
\text { alny w pozycji ,od tyłu”; eufemizm' (MSEP:116) }\end{array}$ \\
\hline \multicolumn{2}{|c|}{ c. Oralverkehr } \\
\hline $\begin{array}{l}\text { geschlossener Kreis - , „69“(Fellatio-Cunnilinctus)“ } \\
\text { (EB:75) }\end{array}$ & $\begin{array}{l}\text { ustny egzamin ,stosunek oralny; eufemizm } \\
\text { zrozumiały jedynie w odpowiednim kontekście' } \\
\text { (MSEP:143 f.) } \\
\text { deep throat, wkładanie penisa podczas stosunku } \\
\text { oralnego aż do gardła; potoczne zapożyczenie } \\
\text { z języka angielskiego' (MSEP:112) } \\
\text { doustny masaż penisa , stosunek oralny; okazjona- } \\
\text { lizm' (MSEP:113) } \\
\text { FOCH ,bardzo odważne pieszczoty oralne; potoczny } \\
\text { eufemizm wulgarny, zrozumiały jedynie w odpo- } \\
\text { wiednim kontekście' (MSEP:115) }\end{array}$ \\
\hline \multicolumn{2}{|c|}{ d. Analverkehr } \\
\hline Schuß ins Schwarze - ,Pedicatio“ (EB:171) & $\begin{array}{l}\text { od tylu ,stosunek doodbytniczy; eufemizm zrozu- } \\
\text { miały jedynie w kontekście' (MSEP:128) }\end{array}$ \\
\hline \multicolumn{2}{|c|}{ e. Homosexueller Geschlechtsverkehr } \\
\hline 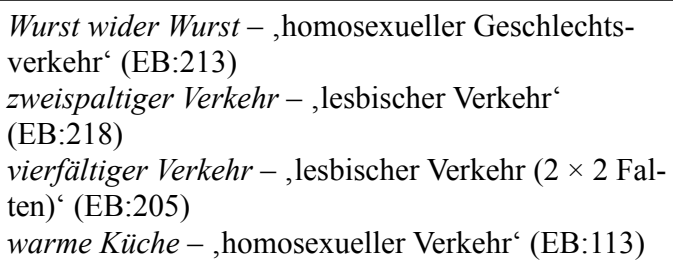 & \\
\hline \multicolumn{2}{|c|}{ f. Geschlechtsverkehr mit mehreren Personen (gleichzeitig) } \\
\hline \multicolumn{2}{|c|}{ - Dreieck } \\
\hline $\begin{array}{l}\text { Klappstulle mit Ei - ,triolistischer Verkehr mit dem } \\
\text { Mann in der Mitte' (EB:104) }\end{array}$ & $\begin{array}{l}\text { jazda na dwa baty ,stosunek seksualny jednej kobie- } \\
\text { ty z dwoma mężczyznami; wulgaryzm' (MSEP:118) }\end{array}$ \\
\hline
\end{tabular}


Klappstulle mit Emmentaler - ,triolistischer Verkehr mit der Frau in der Mitte‘ (EB:104)

Klappstulle mit Riesenbockwurst - , triolistischer Verkehr mit dem Mann, der einen besonders großen Penis besitzt' (EB:104)

Klappstulle mit Schokolade - ,triolistischer Verkehr mit einem Neger oder einer Negerin in der Mitte (EB:104) magiczny trójkąt, stosunek seksualny, w którym mężczyzna odczuwa satysfakcję głównie ze współżycia z dwiema kobietami; okazjonalizm' (MSEP:121)

zabawa we troje, stosunek płciowy polegający na osiąganiu satysfakcji głównie przez kontakty trójosobowe; eufemizm potoczny' (MSEP:148)

\begin{tabular}{|c|c|}
\hline \multicolumn{2}{|c|}{ - Geschlechtsverkehr zu viert } \\
\hline $\begin{array}{l}\text { kleiner Klumpatsch - ,Geschlechtsverkehr zu viert“ } \\
\text { (EB:106) }\end{array}$ & \\
\hline \multicolumn{2}{|c|}{ - Gruppensex } \\
\hline $\begin{array}{l}\text { großer Klumpatsch }-, \text { Geschlechtsverkehr einer } \\
\text { größeren Gruppe‘ (EB:106) } \\
\text { dicke Lotte- }-88 \text { Mitglieder (Gruppensex)‘ (EB:122) } \\
\text { bunte Reihe - ,Sexgruppe beim Gruppensex“ } \\
\text { (EB:153) } \\
\text { Zustände wie im alten Rom - ,Orgien‘ (EB:217) }\end{array}$ & $\begin{array}{l}\text { eksperyment płciowy ,zorganizowanie orgii; } \\
\text { eufemizm okazjonalny’ (MSEP:114) } \\
\text { seksualny happening ,publiczna zabawa o charakte- } \\
\text { rze seksualnym; eufemizm' (MSEP:137) } \\
\text { sex party, przyjęcie połączone z odbywaniem } \\
\text { stosunków seksualnych; eufemizm' (MSEP:137) }\end{array}$ \\
\hline \multicolumn{2}{|c|}{ - Promiskuität (Polyamorie) } \\
\hline & $\begin{array}{l}\text { urzędowanie z wenerycznie chorymi kobietami } \\
\text {,pogardliwie o stosunkach seksualnych z kobietami } \\
\text { chętnie zmieniającymi partnerów; eufemizm’ } \\
\text { (MSEP:143) }\end{array}$ \\
\hline \multicolumn{2}{|c|}{ g. Virtueller Geschlechtsverkehr / Cybersex } \\
\hline & $\begin{array}{l}\text { seks zabawa ,wirtualny seks; potoczne, okazjona- } \\
\text { lizm’ (MSEP:136) }\end{array}$ \\
\hline \multicolumn{2}{|c|}{ h. Betasten } \\
\hline \multicolumn{2}{|l|}{ Tennis des kleinen Mannes - ,Hodenspiel‘ (EB:193) } \\
\hline \multicolumn{2}{|c|}{ i. Geschlechtsverkehr innerhalb der Familie / Inzest } \\
\hline $\begin{array}{l}\text { Liebe im trauten Heim -,Geschlechtsverkehr inner- } \\
\text { halb der Familie“ (EB:87) } \\
\text { im Kreise der Seinigen }-, \text { Kreisorgie mit den } \\
\text { eigenen Kindern' (EB:112) } \\
\text { Stimme des Blutes - ,Neigung zum Inzest' (EB:186) } \\
\text { Glück im Winkel-,Inzest' (EB:212) }\end{array}$ & \\
\hline \multicolumn{2}{|c|}{ j. Anal-Genitalverkehr } \\
\hline $\begin{array}{l}\text { hinten und vorn }- \text {, anal und genital' (EB:91) } \\
\text { vorn und hinten }- \text {, genital und anal' (EB:91) }\end{array}$ & \\
\hline \multicolumn{2}{|c|}{ 2. Masturbieren } \\
\hline \multicolumn{2}{|c|}{ a. sich selbst masturbieren } \\
\hline 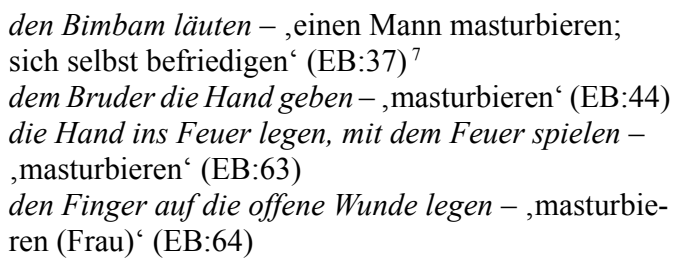 & $\begin{array}{l}\text { jechać na ręcznym - ,masturbować się; potoczne' } \\
\text { (MSEP:159) } \\
\text { samotna rozkosz - ,masturbacja; okazjonalizm' } \\
\text { (MSEP:160) } \\
\text { ktoś bije gruchę / jedzie / strzela / śmiga gruchę- } \\
\text {,o mężczyźnie: ktoś onanizuje się' (SPPW:75) } \\
\text { ktoś trzepie / wali kapucyna - ,o mężczyźnie: ktoś }\end{array}$ \\
\hline
\end{tabular}

7 Abkürzungen in Klammern beziehen sich auf Quellen und Wörterbücher, die am Ende des Beitrags angegeben werden. 
mit der Gitarrespielen-, masturbieren (Frau) ‘(EB:77) die Hand im Spiel haben - ,masturbieren' (EB:83) mit beiden Händen zugreifen - ,zweihändig masturbieren' (EB:84)

die Maus melken - ,masturbieren'(EB:127) jemandem Tribut zollen - , für jemanden masturbieren' (EB:196) onanizuje się' (SPPW:81)

ktoś bije / rabie / trzepie / wali konia - ,o mężczyźnie: ktoś onanizuje się' (SPPW:82)

manipulować przy ptaszku ,o mężczyźnie: masturbować się; potoczne, rubaszne' (MSEP:159)

obkapturzać mnicha - ,o mężczyźnie: masturbować się, potoczne, grubiańskie' (MSEP:159 f.)

trzepać kaszalota - ,o mężczyźnie: masturbować się; wulgarny eufemizm potoczny' (MSEP:160)

\section{b. jemanden masturbieren}

die Wurst abpellen - , einen Mann masturbieren“ (EB:16)

das dritte Ei suchen - ,mit dem Hoden spielen, masturbieren (Mann)' (EB:52)

jemandem die Hand reichen - ,jemanden masturbieren' (EB:83)

die Hände voll zu tun haben - ,jemanden masturbieren, der sich nicht leicht befriedigen läßt' $(\mathrm{EB}: 83)$

mit der Harfe spielen, an der Harfe zupfen, die

Harfe zupfen - , eine Frau masturbieren' (EB:85)

die Harmonika fingern, auf dem Harmonika

klimpfern, mit der Harmonika spielen - ,eine

Frau masturbieren' (EB:85)

das Loch massieren - ,eine Frau masturbieren“

(EB:121)

einem Mann die Stange halten - , ihn masturbieren

(EB:183)

nach den Sternen greifen - ,Männer masturbieren

(EB:185)

er kocht ihr ein Süppchen - ,er masturbiert sie

(EB:190) wytrzepać kapucyna - ,o kobiecie: pieścić ręką penisa aż do osiągnięcia przez partnera pełnej satysfakcji; wulgaryzm potoczny o względnie niskim stopniu nacechowania' (MSEP:147)

\section{Geschlechtsverkehr haben}

mit der Geige geigen - , mit der beischlafwilligen

Freundin coitieren [...]' (WU2: 117)

eine Nummer abziehen - ,koitieren' (EB:18)

den Acker pflügen - ,koitieren' (EB: 18)

den Apparat unter Strom stellen - ,koitieren“ (EB:23)

sie bimmelt pünktlich wie 'n Wecker -, sie hat bei

jedem Koitus prompt einen Orgasmus' (EB:37)

das Brötchen schmieren - ,koitieren' (EB:44)

den Bruder taufen - ,koitieren' (EB:44)

Öl ins Feuer gießen, feuerbohren - ,koitieren“

(EB:63)

auf der G-Saite spielen ,koitieren' (EB:80)

den Kopf ins Loch stecken - ,koitieren' (EB:109)

die Liebesgrotte erforschen - ,koitieren“ (EB:120)

Pfeile schießen - ,mit einer Frau koitieren“ (EB:144)

das Eisen schmieden, so lange es heiß ist - ,koitie-

ren, wenn man Lust hat' (EB:167)

die Trompete polieren - ,koitieren“ (EB:196)

das Vogelhaus lackieren - ,koitieren“ (EB:205) baraszkować z panienkami ,o mężczyźnie: odbywać stosunki seksualne z kobietami; potoczne' (MSEP:108)

borsuczyć sie ,odbyć stosunek seksualny; eufemizm wykorzystujący metaforykę zwierzęcą' (MSEP:108) dawać dupy ,o kobiecie: zgodzić się na stosunek seksualny; pospolity wulgaryzm potoczny' (MSEP:112)

dorwać się do babskiego miodu ,o mężczyźnie: odbyć stosunek seksualny z kobietą; potoczne, rubaszne' (MSEP:113)

grzeszyć / zgrzeszyć z kimś uczynkiem ,mieć z kimś stosunek płciowy. Czasem żartobliwe' (SEP:92)

ktoś zakisit / zamoczyl ogóra ,o mężczyźnie: ktoś odbył stosunek seksualny' (SPPW:106)

nadziać na męski rożen ,o mężczyźnie: odbyć stosunek seksualny; żartobliwy eufemizm, indywidualizm Z. Nienackiego' (MSEP:125)

poruchać serdecznie, odbyć stosunek seksualny; wulgaryzm o wysokim stopniu nacechowania, w tym kontekście nacechowany pozytywnie' (MSEP:131) poznawać sekrety kobiecości ,o mężczyźnie: 


\begin{tabular}{|c|c|}
\hline & $\begin{array}{l}\text { odbywać stosunek seksualny z kobietá' (MSEP:131) } \\
\text { wbijać się } w \text { wilgoć gleby ,o mężczyźnie: odbyć } \\
\text { stosunek seksualny; eufemizm wykorzystujący } \\
\text { ludową metaforykę róslin w mówieniu o sprawach } \\
\text { erotycznych'(MSEP:144) }\end{array}$ \\
\hline \multicolumn{2}{|c|}{ 4. Oralverkehr } \\
\hline 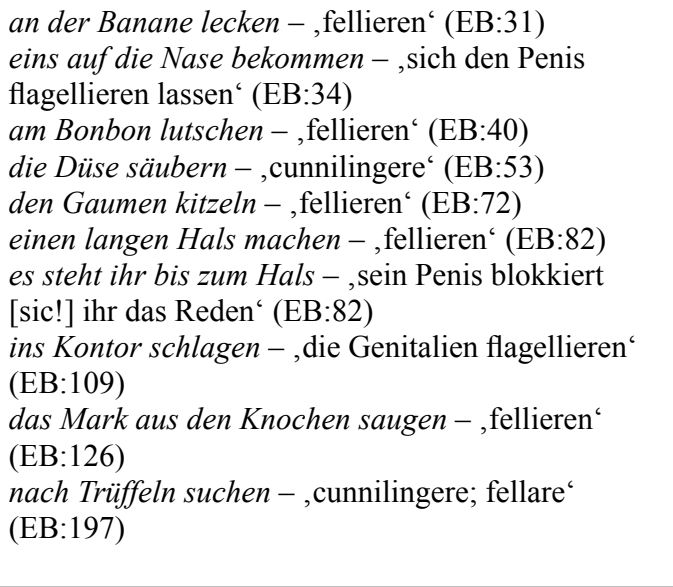 & $\begin{array}{l}\text { tajdaczyć sie po francusku, odbywać stosunek } \\
\text { oralny; eufemizm wulgarny' (MSEP:121) } \\
\text { ciagnać faje ,o kobiecie: odbywać stosunek oralny; } \\
\text { wulgaryzm potoczny' (MSEP:110) } \\
\text { obciagać druta ,o kobiecie: odbyć stosunek oralny; } \\
\text { wulgaryzm o bardzo wysokim stopniu nacechowa- } \\
\text { nia' (MSEP:127) } \\
\text { stawiać gruchę ,o kobiecie: pieścić męski członek aż } \\
\text { do osiągnięcia orgazmu przez mężczyznę; wulga- } \\
\text { ryzm obyczajowy' (MSEP:139) } \\
\text { kochać się języczkiem ,odbyć stosunek oralny; } \\
\text { eufemizm' (MSEP:119) } \\
\text { ktoś dmucha kogoś / komuś w puzon, o partnerze } \\
\text { aktywnym: ktoś drażni ustami i językiem lechtaczkę } \\
\text { i srom, chcąc doprowadzić kobietę do orgazmu' } \\
\text { (SPPW:56) }\end{array}$ \\
\hline \multicolumn{2}{|c|}{ 5. Analverkehr } \\
\hline 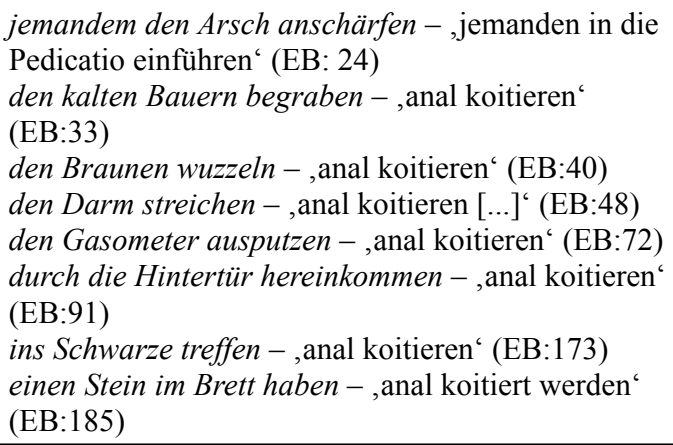 & $\begin{array}{l}\text { dać w dupe ,odbyć z kimś stosunek analny; wul- } \\
\text { garyzm o bardzo wysokim stopniu nacechowania' } \\
\text { (MSEP:112) } \\
\text { posuwać od tyłu,odbyć stosunek analny; wulgaryzm } \\
\text { potoczny' (MSEP:131) } \\
\text { załatwiać od tyłu,odbyć stosunek doodbytniczy; } \\
\text { grubiańskie, potoczne'(MSEP:149) }\end{array}$ \\
\hline \multicolumn{2}{|c|}{ 6. Homosexueller Geschlechtsverkehr } \\
\hline $\begin{array}{l}\text { den Daumen auf den Beutel halten - ,homosexuel- } \\
\text { len Verkehr betreiben; einen Mann masturbieren“ } \\
\text { (EB:49) } \\
\text { einem einen Schlag einhacken - ,einen Mann anal } \\
\text { koitieren' (EB:55) } \\
\text { einen auf die Butten schmeißen - ,einen Mann anal } \\
\text { koitieren' (EB:167) } \\
\text { die Schublade aufbrechen (aufschließen, sprengen } \\
\text { vollpacken) - ,einen Mann anal koitieren“(EB:171) } \\
\text { einem einen stecken - ,einen Mann anal koitieren‘ } \\
\text { (EB:184) }\end{array}$ & $\begin{array}{l}\text { przespać się z przyjacielem ,odbyć stosunek } \\
\text { homoseksualny; eufemizm potoczny’ (MSEP:233) } \\
\text { wyladować z kimś w lóżku ,odbyć przygodny } \\
\text { stosunek seksualny (tu: stosunek homoseksualny); } \\
\text { potoczne' (MSEP:236) }\end{array}$ \\
\hline \multicolumn{2}{|c|}{ 7. Geschlechtsverkehr mit mehreren Personen (gelichzeitig) } \\
\hline \multicolumn{2}{|c|}{ a. Dreieck } \\
\hline $\begin{array}{l}\text { unter einen Hut bringen - ,sich von zwei Männern } \\
\text { gleichzeitig koitieren lassen‘ (EB:96) } \\
\text { siamesisch verkehren - ,mit zwei Männern oder }\end{array}$ & \\
\hline
\end{tabular}


zwei Frauen koitieren (nach den siamesischen

Zwillingen von 1811) (EB:177)

in die Zange nehmen - , beim triolistischen Verkehr

in die Mitte nehmen' (EB:215)

zwei Fliegen mit einer Klappe schlagen - ,zwei

Frauen gleichzeitig befriedigen' (EB:217)

\section{b. Gruppensex}

einen Ball geben - , eine Orgie veranstalten' (EB:31) durch die Bank gehen - , mit einer Gruppe von

Männern koitieren (sowohl hetero- wie homosexuell)' (EB:31)

mehrere Eisen im Feuer haben - , von mehreren

Männern gleichzeitig koitiert werden' (EB:56)

durch die Schnur gehen - , mit allen anwesenden

Partnern koitieren (Gruppensex) ' (EB:169)

Spalier laufen - , mit allen anwesenden Männern koitieren (Gruppensex)` (EB:179)

\section{Betasten}

unter den Arm greifen - ,einem Mann an die Hoden fassen' (EB:24)

die Augen in die Hand nehmen - , die Brüste

berühren' (EB:27)

ei ei machen - ,streicheln, liebkosen' (EB:54)

ihr Herz in die Hand nehmen - ,ihren Busen

auffassen' (EB:89)

die dritte Klöte suchen - ,mit den Hoden spielen“

(EB:105)

die Kirchturmglocken läuten - , mit den Hoden spielen' (EB:117)

ins Volle greifen - , an den Busen fassen' (EB:206)

\section{Anale Masturbation}

im Bergwerk krabbeln - , anal koitieren, anal masturbieren' (EB:35)

die schwarze Kammer auswichsen - ,im Anus

masturbieren' (EB:99)

den Schlauch ausbeuteln - ,im After masturbieren“

(EB: 165)

im Tunnel rangieren - ,im After masturbieren

(EB:198)

am Zwetschenkern zupfen - ,im After masturbieren“

(EB:218)

\section{Geschlechtsverkehr im Bordell/mit Prostituierten}

am Eckstein stempeln - ,mit einem Straßenmädchen

koitieren' (EB:54, 185)

einen Bolzen stemmen - ,mit einer Prostituierten

schlafen' (EB:185)

\section{Geschlechtsverkehr innerhalb der Familie / Inzest}

seinen eigenen Kohl bauen - , seinen Sexualverkehr

auf Mitglieder der eigenen Familie beschränken`

(EB:108)

auf eigener Scholle säen - ,mit seinen Kindern

koitieren' (EB:169) 


\begin{tabular}{|c|c|}
\hline \multicolumn{2}{|c|}{ 12. Anal-Genitalverkehr } \\
\hline $\begin{array}{l}\text { auf den Damm gehen - , gleichzeitig anal und genital } \\
\text { koitieren oder koitiert werden“ (EB:48) } \\
\text { die Köpfe zusammenstecken - ,eine Frau gleich- } \\
\text { zeitig genital und anal koitieren' (EB:110) }\end{array}$ & \\
\hline \multicolumn{2}{|c|}{ 13. Anal-Oralverkehr } \\
\hline $\begin{array}{l}\text { Braunkohl ausheben - , anilingere‘ (EB:41) } \\
\text { den Keller säubern }- \text {, anilingere‘ (EB:101) } \\
\text { am Mostrich naschen - ,Kot essen, anilingere‘ } \\
\text { (EB:131) } \\
\text { die Rosette küssen -, anilingere‘ (EB:156) } \\
\text { die Zwetsche polieren - , anilingere‘ (EB:218) }\end{array}$ & \\
\hline \multicolumn{2}{|c|}{ 14. Penis-Penis-Verkehr } \\
\hline $\begin{array}{l}\text { Kopf an Kopf stehen - ,mit einem Penis den anderen } \\
\text { berühren' (EB:110) }\end{array}$ & \\
\hline \multicolumn{2}{|c|}{ 15. Busen-Sex } \\
\hline 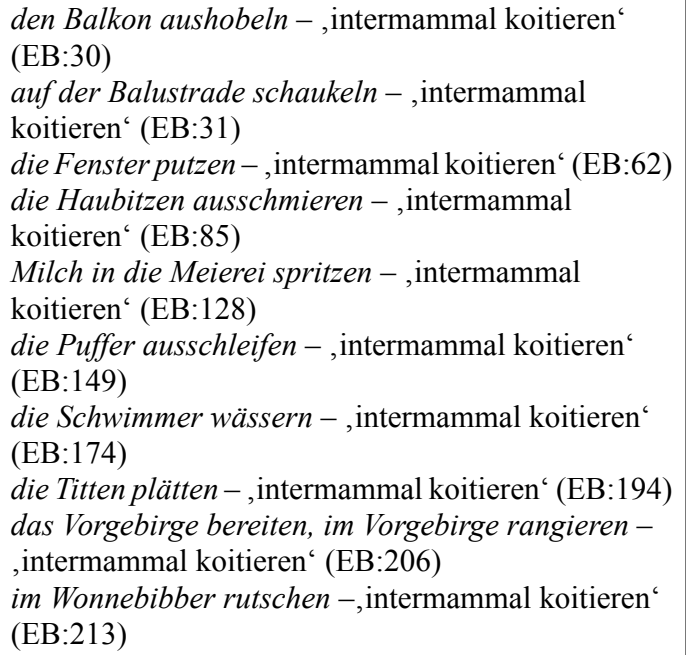 & \\
\hline \multicolumn{2}{|c|}{ 16. Promiskuität (Polyamorie) } \\
\hline & $\begin{array}{l}\text { tajdaczyć się, odbywać stosunki seksualne z } \\
\text { różnymi partnerami; potoczne' (MSEP:120) } \\
\text { puszczać sie ,o kobiecie: odbywać często stosunki } \\
\text { seksualne z różnymi partnerami; wulgaryzm potocz- } \\
\text { ny o niskim stopniu nacechowania' (MSEP:134) } \\
\text { trwonić nasienie po wiejskich dziewuchach,od- } \\
\text { bywać stosunki seksualne z wieloma partnerkami; } \\
\text { okazjonalizm' (MSEP:142) } \\
\text { wskoczyć do tóżka ,o kobiecie: często i chętnie } \\
\text { odbywać stosunki seksualne z nowymi partnerami; } \\
\text { eufemizm' (MSEP:146) } \\
\text { zaliczać kogoś ,o kobiecie: odbywać częste } \\
\text { stosunki seksualne z różnymi partnerami; eufemizm } \\
\text { potoczny' (MSEP:149) }\end{array}$ \\
\hline
\end{tabular}

Tab. 1. Deutsche und polnische Phraseologismen zur Beschreibung der Geschlechtsverkehrstypen 


\section{Schlussfolgerungen}

Aufgrund des dargestellten Materials kann man feststellen, dass sich sowohl deutsche als auch polnische Phraseologismen aus dem Bereich der Sexualität auf diverse Geschlechtsverkehrsarten beziehen.

Beispiele, die Masturbieren beschreiben, lassen sich in beiden Sprachen finden. Darunter werden sowohl die Selbstbefriedigung als auch die Befriedigung anderer Personen unterschieden. Einheiten, die sich auf Tiere beziehen, sind sowohl im Deutschen als auch im Polnischen vorhanden, z. B.: die Maus melken, manipulować przy ptaszku und trzepać kaszalota. Im Falle solcher Beispiele kann man auch bemerken, dass die Genitalien mit Namen der Tiere bezeichnet werden. Deutsche Beispiele wecken Assoziationen u. a. mit Instrumenten (z. B.: die Harmonika fingern, auf dem Harmonika klimpfern, mit der Harmonika spielen und mit der Harfe spielen, an der Harfe zupfen, die Harfe zupfen) und Essen (z. B.: die Wurst abpellen und er kocht ihr ein Süppchen). Die polnischen Beispiele beziehen sich u. a. auf die Ähnlichkeit der Bewegung beim Masturbieren und bei der Handbremse in einem Auto, z. B.: jechać na ręcznym. Überdies kann man im Polnischen auf die Wendung obkapturzać mnicha hinweisen. Hier wird das Aussehen des Mönchs (Kapuze und Kahlkopf) mit dem Aussehen des Penis (Vorhaut und Glans Penis) verglichen.

Die Gruppe Geschlechtsverkehr enthält die größte Anzahl der Beispiele sowohl im Deutschen als auch im Polnischen. In beiden Sprachen wird der Bereich Essen thematisiert z. B.: das Brötchen schmieren und ktoś zakisit / zamoczyl ogóra. Hier werden die Genitalnamen mithilfe von Lebensmittelnamen ersetzt. Die deutschen Wendungen beziehen sich auf Musik bzw. Instrumente, das sind u. a. mit der Geige geigen, auf der G-Saite spielen und die Trompete polieren. Eines der interessanten Beispiele ist auch den Apparat unter Strom stellen, das Bezug auf die Verbindung eines Geräts zum Laden nimmt, was mit einem Geschlechtsverkehr assoziiert werden kann. Im Polnischen kann man Entlehnungen aus der Tierwelt finden, z. B.: borsuczyć się. Das Beispiel poruchać serdecznie präsentiert eine interessante Verbindung, weil das Verb ruchać bzw. poruchać negativ geladen ist. Es wurde aber mit dem Adverb serdecznie zusammengestellt. Dank dieser Verbindung wird die ganze Phrase gemildert und hat keinen so stark negativen Beiklang mehr.

In der Gruppe Oralverkehr kann man auch auf solche Bereiche hinweisen, die in den beiden Sprachen thematisiert werden, u. a. Essen bzw. Lebensmittel (z. B.: an der Banane lecken, am Bonbon lutschen, nach Trüffeln suchen und stawiać gruchę). Auch in dieser Gruppe werden Genitalien mithilfe von Lebensmitteln genannt. Sowohl im Polnischen als auch im Deutschen kann man Beispiele finden, die sich auf das Organ beziehen, das das Schlüsselorgan beim Oralverkehr ist, nämlich Hals bzw. Mund, und zwar einen langen Hals machen, es steht ihr bis zum Hals, tajdaczyć się po francusku und obciagać druta.

Man kann bemerken, dass das Beispiel deep throat, das sich im polnischen Wörterbuch finden ließ, eine Entlehnung aus dem Englischen ist. Das deutsche Beispiel geschlossener Kreis bezieht sich auf den zweiseitigen Oralverkehr und visualisiert die Sexposition 69, die wie ein Kreis aussieht. Im Polnischen soll man auf die Abkürzung FOCH aufmerksam machen, deren volle Form Fachowe Obciaganie Chuja ist und eine vulgäre Komponente enthält.

Idiomatische Wendungen, die sich auf den Analverkehr konzentrieren, enthalten in beiden Sprachen Lexeme oder Metaphern, die sich auf den Hintern beziehen, z. B.: den Braunen wuzzeln, durch die Hintertür hereinkommen, dać $w$ dupę und od tyłu. Im Deutschen kann man auch einen Phraseologismus finden, dessen Bedeutung in die sexuelle Bedeutung umgewandelt wurde, nämlich ins Schwarze treffen, wobei das Schwarze für Anus steht.

Es gibt auch Einheiten, die den homosexuellen Verkehr zum Ausdruck bringen. In dieser Gruppe kann man aber ein Missverhältnis zwischen der Kreativität der analysierten Sprachen beobachten. Im Deutschen beziehen sich die Beispiele auf die Geschlechtsorgane, die von anderen Lexemen ersetzt werden, z. B.: Wurst wider Wurst und zweispaltiger Verkehr. In polnischen Beispielen wird der homosexuelle Verkehr nur in den Wörterbuchdefinitionen angegeben. Die Beispiele selbst wecken keine Assoziationen mit diesem Geschlechtsverkehrstyp. 
Es gibt auch Phraseme, die Bezug auf Geschlechtsverkehr mit mehreren Personen nehmen. Darunter kann man den Geschlechtsverkehr zu dritt, zu viert und Gruppensex unterscheiden. In allen Untergruppen ist die Disproportion zwischen den analysierten Sprachen sichtbar, wenn es um Kreativität und die Wortschatzmenge geht. In der Gruppe Dreieck soll man auf das deutsche Beispiel zwei Fliegen mit einer Klappe schlagen hinweisen, der die Bedeutung auf den sexuellen Bereich überträgt. In den polnischen Beispielen kann man beobachten, dass das Lexem trójkąt verwendet wird, das auf die Anzahl der am Geschlechtsverkehr teilnehmenden Personen hinweist. In der Untergruppe $z u$ viert gibt es nur ein deutsches Beispiel, nämlich kleiner Klumpatsch. Es gibt keine polnischen Beispiele, die sich auf den Geschlechtsverkehr zu viert beziehen. In der Untergruppe Gruppensex kann man Beispiele in beiden Sprachen finden. Im Deutschen lassen sich Beispiele unterscheiden, die die Vorstellungskraft der Menschen bildlich beeinflussen oder sich auf alte Zeiten beziehen, z. B.: mehrere Eisen im Feuer haben und Zustände wie im alten Rom. Im Polnischen gibt es u. a. Beispiele, die Bezug auf Partys bzw. Veranstaltungen nehmen, z. B.: seksualny happening und sex party.

Darüber hinaus kann man auf verschiedene Beispiele hinweisen, die nur in der deutschen Sprache vorkommen und diverse Geschlechtsverkehrstypen beschreiben. Darunter befindet sich u. a. Betasten. Hier gibt es Beispiele mit der Komponente Hand, z. B.: die Augen in die Hand nehmen und ihr Herz in die Hand nehmen. Man kann auch verschiedene Typen von Masturbation unterscheiden, d. h. eine gegenseitige Masturbation von Männern, die mit dem Händedrück assoziiert wird, z. B.: ein Händedruck unter Männern, und eine anale Masturbation, wobei das Hintern bzw. der Anus durch andere Lexeme ersetzt wird, z. B.: im Tunnel rangieren.

Im Deutschen gibt es auch Beispiele, die sich auf den Geschlechtsverkehr in einem Bordell beziehen (einen Bolzen stemmen) oder innerhalb einer Familie (Liebe im trauten Heim). Man soll darauf aufmerksam machen, dass das Beispiel Liebe im trauten Heim einen euphemistischen Charakter aufweist.

Man kann auch deutsche Einheiten finden, die den Anal-Genitalverkehr, Anal-Oralverkehr, Penis-Penis-Verkehr und Busen-Sex beschreiben. In all diesen Gruppen ist es charakteristisch, die Geschlechtsorgane, den Hintern und die Körperteile metaphorisch zu benennen, d. h.: hinten und vorn, die Rosette küssen, Kopf an Kopf stehen und den Balkon aushobeln.

Es gibt auch einige Gruppen, die nur in der polnischen Sprache zu finden sind. Die polnischen Beispiele beschreiben auch den Geschlechtsverkehr mit mehreren Personen, aber es geht nicht um den gleichzeitigen Verkehr, sondern um ausschweifendes Leben. Darunter befinden sich folgende idiomatische Einheiten: kurwić się, puszczać się und trwonić nasienie po wiejskich dziewuchach. Darüber hinaus wird Cybersex im Polnischen auf idiomatische Art und Weise zum Ausdruck gebracht. Hier kann man u. a. das folgende Beispiel angeben: seks zabawa.

Zusammenfassend kann man feststellen, dass sich sowohl in deutschen als auch in polnischen Wörterbüchern viele Beispiele auf die Ähnlichkeit der Geschlechtsorgane mit anderen Gegenständen beziehen. Man kann auch bemerken, dass fast alle Wendungen aus vielen Alltagsbereichen in einem bestimmten Kontext und mit einer bestimmten Intention eine sexuelle Bedeutung tragen können. In diesem Zusammenhang kann man vor allem darauf aufmerksam machen, dass sich viele Beispiele auf das Essen bzw. Lebensmittel, die Musik und Instrumente oder die Tierwelt beziehen. In deutschen Nachschlagewerken sind dagegen viele Phraseologismen zu finden, deren primäre Bedeutung um die Bedeutung aus dem sexuellen Bereich erweitert wird. Überdies lässt es sich beobachten, dass in den untersuchten deutschsprachigen lexikalischen Werken mehrere vielfältigere und kreativere Wendungen enthalten sind und diese mehreren idiomatischen Wendungen bieten, die Sex beschreiben. Auf der anderen Seite beobachtet man viele phraseologische Einheiten, die eine wörtliche Lesart haben, die aber in Bezug auf Sexualität eine völlig neue Bedeutung gewinnen.

\section{Literaturverzeichnis}

\section{Primärliteratur:}

EB = BORNEMAN, Ernest (2013): Sex im Volksmund. Der obszöne Wortschatz der Deutschen. Berlin. 
MSEP = KOCIEMBA-ŻULICKA, Justyna (2011): Mały słownik erotyzmów polskich. Ostrów Wielkopolski.

SEP = DĄBROWSKA, Anna (2013): Stownik eufemizmów polskich, czyli w rzeczy mocno, $w$ sposobie tagodnie. Warszawa.

SPPW = GROCHOWSKI, Maciej (1995): Stownik polskich przekleństw i wulgaryzmów. Warszawa. WU2 = KÜPPER, Heinz (1966): Wörterbuch der deutschen Umgangssprache. Bd. 2. Hamburg.

\section{Sekundärliteratur:}

BĄK, Paweł (2012): Euphemismen des Wirtschaftsdeutschen aus Sicht der anthropozentrischen Linguistik. Frankfurt a. M.

Burger, Harald (2015): Phraseologie. Eine Einführung am Beispiel des Deutschen. Berlin.

DąBrowsKa, Anna (1994): Eufemizmy współczesnego języka polskiego. Wrocław.

DĄBRowsKa, Anna (2006): Eufemizmy współczesnego języka polskiego. Wrocław.

DietL, Cora (1996): Euphemismus. In: UEDING, Gert (Hrsg.): Historisches Wörterbuch der Rhetorik, Bd. 3. Tübingen, S. 1-10.

DiETZ, Hans-Ulrich (1999): Rhetorik in der Phraseologie: zur Bedeutung rhetorischer Stilelemente im idiomatischen Wortschatz des Deutschen. Tübingen.

FLEISCHER, Wolfgang (1982): Phraseologie der deutschen Gegenwartssprache. Leipzig.

FLEISCHER, Wolfgang (1997): Phraseologie der deutschen Gegenwartssprache. Tübingen.

ForSTER, Iris (2009): Euphemistische Sprache im Nationalsozialismus: Schichten, Funktionen, Intensität. Bremen.

Frietsch, Ute / Hanitzsch, Konstanze /John, Jennifer / Beatrice, Michaelis (Hrsg.) (2007): Geschlecht als Tabu: Orte, Dynamiken und Funktionen der De/Thematisierung von Geschlecht. Bielefeld.

Knaut, Tina (2011): Berichterstattung über Sexualität: Zwischen Tabuisierung und Pornografisierung Eine Literaturanalyse zum gegenwärtigen Stand der Diskussion. Hamburg.

Nefedova, Ljubov (2019): Höfliche Unhöflichkeit im Deutschen und Russischen. In: GONDEK, Anna / SZCZĘK, Joanna (Hrsg.): Deutsche Phraseologie und Parömiologie der (Un)Höflichkeit, Sektionsbeiträge der internationalen EUROPHRAS- Tagung in Białystok/Polen, 10.-12. September 2018. Hamburg, S. 283-297.

Nickel, Volker (2003): Das Tabu in der Werbung. In: Depenheuer, Otto (Hrsg.) Recht und Tabu. Wiesbaden.

Palm, Christine (1997): Phraseologie: Eine Einführung. Tübingen.

RADA, Roberta (2013): Tabus und Euphemismen in der deutschen Gegenwartssprache: mit besonderer Berücksichtigung der Eigenschaften von Euphemismen. Budapest.

Schuch, Hans Waldemar (2012): Auflösungserscheinungen und Normalisierungseinpflegungen Reden über den Sex. In: Integrative Therapie, Vol 38. No. 2 (Oktober 2012). Wien, S. 103-166.

WAŚNIEWSKA, Małgorzata (2017): 'Misja pokojowa' czy 'krwawa jatka': eufemizmy i dysfemizmy $\mathrm{w}$ dyskursie medialnym na przykładzie relacji z wojny w Syrii w polskiej prasie. In: tekst $i d y s-$ kurs - text und dyskurs 10, 2017.

\section{Internetquellen}

URL 1: Lauenstein, Mercedes (2017): Nicht jedes Tabu muss gebrochen werden! Denn sie können uns auch schützen. https://www.jetzt.de/gutes-leben/wie-wichtig-sind-tabus (11.11.2017) [22.04.2020].

URL 2: https://www.duden.de/rechtschreibung/Tabu [22.4.2020].

URL 3: https://www.ikud.de/glossar/tabu-begriffsherkunft-definition.html [22.4.2020].

URL 4: https://www.duden.de/rechtschreibung/Euphemismus [23.4.2020]. 